\title{
Estrogen enhances the bone regeneration potential of periodontal ligament stem cells derived from osteoporotic rats and seeded on nano-hydroxyapatite/collagen/poly(L-lactide)
}

\author{
LING-LING E ${ }^{1 *}$, WEN-HUAN XU ${ }^{2 *}$, LIN FENG ${ }^{1}$, YI LIU $^{1}$, DONG-QING CAI ${ }^{3}$, NING WEN ${ }^{1}$ and WEN-JIE ZHENG ${ }^{3}$ \\ ${ }^{1}$ Institute of Stomatology, ${ }^{2}$ Scientific Research Department, Medical Administrative Division, Chinese PLA General Hospital, \\ Beijing 100853; ${ }^{3}$ Department of Chemistry, Jinan University, Guangzhou, Guangdong 510632, P.R. China
}

Received July 21, 2015; Accepted March 28, 2016

DOI: 10.3892/ijmm.2016.2559

\begin{abstract}
This study investigated the effects of estrogen on the bone regeneration potential of periodontal ligament stem cells (PDLSCs) derived from osteoporotic rats and seeded on a collagen-based composite scaffold [nano-hydroxyapatite/collagen/poly(L-lactide) (nHAC/PLA)]. For this purpose, 48 healthy 3-month-old Sprague-Dawley female rats were divided into 2 groups as follows: the bilaterally ovariectomized (OVX) rats and sham-operated rats. The PDLSCs were isolated at 3 months after surgery (by which time postmenopausal osteoporosis had developed). The effects of estrogen on the characteristics of these cells seeded in a culture plate and of the cells seeded on nHAC/PLA were then investigated. The PDLSC + nHAC/PLA constructs were implanted subcutaneously into the backs of severe combined immunodeficient (SCID) mice for 12 weeks in order to examine the role of estrogen in the bone formation ability of PDLSCs derived from osteoporotic rats. The results from methyl thiazolyl tetrazolium (MTT) assay revealed that the proliferation of the cells derived from the rats in the OVX group was significantly higher than that of the cells derived from the rats in the sham-operated group at the stage of logarithmic growth. The staining intensity of alkaline phosphatase (ALP) and the mineralization of the cells derived from the rats in the OVX group was significantly weaker than that of the cells from the rats in the sham-operated group. When the PDLSCs were seeded on nHAC/PLA, ALP activity, osteocalcin (OCN) secre-
\end{abstract}

Correspondence to: Dr Ning Wen, Institute of Stomatology, Chinese PLA General Hospital, 28 Fuxing Road, Beijing 100853, P.R. China E-mail: wenningchn@163.com

Dr Wenjie Zheng, Department of Chemistry, Jinan University, 601 Huangpu Avenue West, Guangzhou, Guangdong 510632, P.R. China

E-mail: tzhwj@jnu.cn

*Contributed equally

Key words: periodontal diseases, postmenopausal osteoporosis, periodontal ligament stem cells, ovariectomy, estrogen, nanohydroxyapatite/collagen/poly(L-lactide) tion, mineral formation and the mRNA expression levels of ALP, $\mathrm{OCN}$, estrogen receptor (ER) $\alpha$ and ER $\beta$ in the cells derived from the rats in the OVX group were markedly decreased. Treatment with $17 \beta$-estradiol (E2) significantly weakened the proliferative ability of the cells derived from the OVX group rats, and enhanced their osteogenic differentiation ability and the mRNA expression levels of ALP, OCN, ER $\alpha$ and ER $\beta$. When the constructs were implanted into the backs of SCID mice for 12 weeks, the results of histological analysis indicated that the constructs derived from the OVX group rats had a few newly formed bones and osteoids; however, a great number of newly formed bones and osteoids were present in the ones from the sham-operated group and the OVX + E2 group rats. Our findings further indicate that estrogen deficiency impairs the osteogenic differentiation potential of PDLSCs, and that ER plays an important role in the bone regeneration ability of PDLSCs. Estrogen enhances the bone regeneration potential of PDLSCs derived from osteoporotic rats and seeded on nHAC/PLA. This study may provide insight into the clinical management of periodontal bone tissue repair in postmenopausal women with the use of estrogen-mediated PDLSCs seeded on nHAC/PLA.

\section{Introduction}

Estrogens are hormones that are important for sexual and reproductive development, mainly in women. Estrogen is instrumental in bone formation, and together with vitamin D, calcium and other hormones, it effectively breaks down and rebuilds bones according to the body's natural processes. As estrogen levels begin to decline in middled-aged women, the process of and the ability to rebuild bone dimishes. Consequently, in postmenopausal women, the amount of bone being broken down excedes that of bone being rebuilt (1).

Estrogen deficiency, which is vital in the pathogenesis of postmenopausal osteoporosis (2), has received increasing attention in studies examining periodontal diseases. There have been reports of periodontitis-associated bone loss in the mandibular body in ovariectomized (OVX) animals (3-6); clinical observations in postmenopausal women have confirmed an increased prevalence of periodontal disease with low estrogen levels (7-11). Symptoms, such as thinning of the mandibular inferior cortex $(12,13)$ and residual ridge reduction of the 
edentulous jaw $(14,15)$ have been reported in patients with postmenopausal osteoporosis, which suggest a link between postmenopausal osteoporosis and the loss of periodontal tissue.

Conventional periodontal tissue regenerative therapies, such as guided tissue regeneration, depending on the individual anatomy of the defects or the amount of resident vital periodontal ligament, can partially regenerate periodontal tissues (16-21). Until now, the most ideal strategy of periodontal tissue regeneration therapies is to control inflammation and stimulate stem progenitors to regenerate new periodontal tissues. The periodontal ligaments are very important in maintaining the integrity of the periodontal tissue, which is the connective tissue located between the alveolar bone and the root surface of the tooth. The periodontal ligament stem cells (PDLSCs) of this ligament exhibit osteoblast-like characteristics and are capable of differentiating into cementoblasts or osteoblasts, and can regenerate new functional periodontal support tissues, including the cementum, the alveolar bone and the periodontal ligament fiber $(22,23)$, and are the most ideal seed cells of periodontal tissue regeneration.

Nowadays, bone grafting procedure limitations have shifted the focus of preclinical research to tissue engineering strategies, which are strictly dependent on the proliferation and differentiation ability of mesenchymal stem cells (MSCs); however, they may be affected by genetics, aging, hormone levels, drug consumption, or chronic systemic disease (24). MSCs have the ability to self-renew, proliferate and differentiate toward different lineages (osteoblasts, adipocytes, chondroblasts and myoblasts); however, MSCs derived from bone marrow (BM-MSCs), which are the most commonly employed cells in the clinical setting for different orthopedic diseases (25), are reported to be negatively influenced by various factors, most of which are also responsible for osteoporosis (26), such as systemic diseases, lifestyle, drug consumption and aging.

Several studies have also investigated the association between changes in levels of the female sex hormone, estrogen, and changes in PDLSCs (27,28), which modulates the activity of PDLSCs by binding to the intracellular estrogen receptor (ER) (29-31). Zhang et al (32) also reported that estrogen deficiency leads to the impaired osteogenic differentiation of PDLSCs in osteoporotic rats. Nonetheless, the mechanisms responsible for the effects of estrogen on PDLSCs are poorly understood. It is important to clarify the biological function of the female sex hormone, estrogen, in PDLSCs, as this hormone may affect periodontal health. Moreover, the effects of estrogen on the bone regeneration potential of PDLSCs isolated from osteoporotic rats in vivo have not yet been fully elucidated. Therefore, it remains undetermined as to whether functional periodontal tissue can be regenerated following the transplantation of autologous PDLSCs isolated from osteoporotic animals.

The nano-hydroxyapatite/collagen/poly(L-lactide) (nHAC/ PLA), a three-dimensional (3D) nanostructured scaffold, is an attractive bone substitute, as the novel biomimetic strategy used to generate this scaffold endows it with properties similar to those of natural bone. Cell culture and animal model experiments have demonstrated that the composite material is highly osteoconductive, biocompatible and bioresorbable $(33,34)$.

In the present study, we created an estrogen deficiency microenvironment by ovariectomy and examined the effects of estrogen deficiency and estrogen supplementation on the bone-forming capacity of PDLSCs derived from osteoporotic rats and seeded on $\mathrm{nHAC/PLA}$, both in in vitro and in vivo. This may provide an ideal approach for functional periodontal tissue regeneration in postmenopausal women with periodontal disease.

\section{Materials and methods}

Establishment of the animal model of osteoporosis. All surgical procedures and care administered to the animals were approved by the University Animal Care Committee and performed according to institutional guidelines. A total of 48 healthy 3-month old Sprague-Dawley (SD) female rats (Experimental Animal Center of the Academy of Military Medical Sciences) were randomly divided into 2 groups as follows: 24 animals were in the bilaterally ovariectomized (OVX) group, and the other 24 animals were subjected to sham surgery (shamoperated group, hereinafter referred to as the sham group). In the sham procedure, the ovaries were exteriorized and replaced intact to create a stress similar to that obtained with bilateral ovariectomy, in accordance with the method described in the study by Yu et al (35).

Measurement of body weight, bone mineral density (BMD) and the estrogen level. At 0 (baseline) and 3 months after surgery, the SD rats from the 2 groups were anesthetized with $10 \%$ chloral hydrate $(400 \mathrm{mg} / \mathrm{kg})$ and weighed; blood samples were obtained from the retro-orbital plexus to evaluate the estrogen level. The BMD of the lumbar spine was measured using a DEXA scanner (GE Healthcare Lunar; GE Healthcare, Madison, WI, USA). To measure the lumbar spine BMD, the rats were placed in the prone position on the DEXA plate and the X-ray tube was aligned along the longitudinal axis at the mid-point of the body. The BMD value was determined using small animal analysis software (GE Lunar Prodigy, Prodigy enCORE software version 10.50.086; GE Healthcare).

Cell culture and isolation of PDLSCs. The rat PDLCs were isolated in accordance with the method described in the study by Gay et al (36). Briefly, the sham-operated and ovariectomized SD rats after 3 months of surgery were sacrificed for gathering the periodontal ligament. The PDLCs were collected from the periodontal ligament which were rinsed and cut into trivial blocks under sterile conditions and digested in $0.3 \%$ collagenase (Invitrogen, Carlsbad, CA, USA) at $37^{\circ} \mathrm{C}$ for $4 \mathrm{~h}$ on a rotator set at $130 \mathrm{rpm}$. The cells otained were then seeded in a $75 \mathrm{~cm}^{2}$ culture flask and cultured in growth medium (GM) containing Dulbecco's modified Eagle's medium (DMEM), $10 \%$ fetal bovine serum (FBS), $100 \mathrm{IU} / \mathrm{ml}$ penicillin and $100 \mathrm{IU} / \mathrm{ml}$ streptomycin (all from Invitrogen) in a humidified atmosphere $\left(95 \%\right.$ air, $\left.5 \% \mathrm{CO}_{2}\right)$ at $37^{\circ} \mathrm{C}$. The cells were fed every 3 days with GM. After the cells reached confluence, they were trypsinized and plated in the culture flasks. Subsequently, PDLSCs were obtained as previously described (37). Briefly, STRO- $1^{+}$stem cells were isolated using immunomagnetic beads (Dynabeads; Dynal Biotech, Oslo, Norway) according to the manufacturer's instructions. After washing, bead-positive cells were segregated using a magnetic particle separator and subsequently seeded into a $75 \mathrm{~cm}^{2}$ culture flask and cultured in $\mathrm{GM}$ in a humidified atmosphere $\left(95 \%\right.$ air, $\left.5 \% \mathrm{CO}_{2}\right)$ at $37^{\circ} \mathrm{C}$. PDLSCs at passage 4 were used in each experiment. At least 3 replicates were included for analysis. 
Phenotypic analysis of PDLSCs. The PDLSCs at passage 4 isolated from the rats in the sham group and OVX group were seeded into chamber slides, cultured for 7 days in GM, fixed with cold acetone for $10 \mathrm{~min}$ and immunostained for confocal laser scanning microscopy (CLSM). The cells were blocked and permeabilized $1 \mathrm{~h}$ at room temperature in Tris-buffered saline solution (TBS), $\mathrm{pH} 7.4$, containing $10 \%$ FBS, $1 \%$ bovine serum albumin (BSA) and 0.5\% Triton X-100 (Sigma Chemical Co., St. Louis, MO, USA). Antibody labeling was performed overnight at room temperature [vimentin 1:100 (MAB2105), keratin 1:100 (MAB3165); R\&D Systems, Minneapolis, MN, USA]. Secondary antibodies were applied for $2 \mathrm{~h}$ at room temperature [anti-mouse IgG TRITC 1:50 (sc-3796); Santa Cruz Biotechnology, Inc., Santa Cruz, CA, USA]. Following repeated washes with phosphate-buffered saline (PBS), nuclear staining was performed with 4',6-diamidino-2-phenylindole (DAPI; Sigma Chemical Co.) at room temperature for $15 \mathrm{~min}$, and Mowiol 4-88 confocal images were recorded using a Zeiss LSM 5 Pascal system with a Zeiss Axiovert microscope (Carl Zeiss, Oberkochen, Germany).

Immunohistochemistry for STRO-1 was performed on the stem cells isolated from the 2 groups of rats cultured on chamber slides for 7 days. The cells were fixed with $10 \%$ formalin, and were then blocked and permeabilized $1 \mathrm{~h}$ at room temperature in TBS, $\mathrm{pH} 7.4$, containing $10 \%$ FBS, $1 \%$ BSA and $0.5 \%$ Triton X-100. The cells were then incubated with mouse antibodies against human STRO-1 (1:100, MAB1038) (R\&D Systems) overnight at $4^{\circ} \mathrm{C}$. The cells were then washed with PBS and incubated with biotin-conjugated swine-anti-mouse antibodies (sc-2031; Santa Cruz Biotechnology, Inc.) for $2 \mathrm{~h}$, washed in PBS and incubated with streptavidin-biotin complex/horseradish peroxidase (Santa Cruz Biotechnology, Inc.) for $1 \mathrm{~h}$. Staining was visualized using 3,3'-diaminobenzidine (DAB, $0.1 \mathrm{mg} / \mathrm{ml}, 0.02 \% \mathrm{H}_{2} \mathrm{O}_{2}$ ) (Santa Cruz Biotechnology, Inc.).

Methyl thiazolyl tetrazolium (MTT) assay. MTT assay was performed as previously described in the study by $\mathrm{E}$ et al (38). For the experiment, the cells were divided into 3 groups as follows: the sham group, the OVX group and the OVX $+17 \beta$-estradiol (E2) group. Briefly, the cells at passage 4 were plated in 96 -well plates at $2 \times 10^{4}$ cells $/ \mathrm{ml}$ with $200 \mu \mathrm{l} \mathrm{GM}$ for $24 \mathrm{~h}$ to allow attachment. The serum-containing medium was then removed and replaced with a medium without FBS for a further $12 \mathrm{~h}$. The cells in the sham group and OVX group were cultured in GM, and the cells in the OVX + E2 group were cultured in $\mathrm{GM}+10^{-7} \mathrm{M}$ E2 (Sigma Chemical Co.). Following culture for days 1,2,3, 4,5,6,7 and $8,20 \mu 15 \mathrm{mg} / \mathrm{ml}$ MTT solution/well was added to the cells, followed by culture for $4 \mathrm{~h}$. The cells were washed twice with PBS, and $150 \mu \mathrm{l}$ dimethyl sulfoxide (Sigma Chemical Co.) was then added to each well, followed by shaking for $10 \mathrm{~min}$, and the optical density values for each well were determined at $490 \mathrm{~nm}$.

Cell culture protocol for histochemical analysis of alkaline phosphatase (ALP) activity and mineralization. For the experiment, the cells were divided into 3 groups: the sham group, the OVX group and the OVX + E2 group. Briefly, the cells at passage 4 were plated at $1 \times 10^{5}$ cells $/ \mathrm{ml}$ in 6-well culture plates with GM for $24 \mathrm{~h}$ to allow attachment. The serum-containing medium was then removed and replaced with a medium without
FBS for a further $12 \mathrm{~h}$. The cells in the sham group and OVX group were cultured in an osteogenic medium (ODM) [growth medium containing $10 \mathrm{nM}$ dexamethasone, $50 \mathrm{mg} / \mathrm{ml}$ ascorbic acid and $100 \mathrm{mM} \beta$-glycerophosphate (Sigma Chemical Co.)], and the cells in the OVX + E2 group were cultured in $\mathrm{ODM}+10^{-7} \mathrm{M}$ E2. For the histochemical analysis of ALP activity and mineralization, the cells were cultured for 21 days. The medium was replaced every 4 days.

To determine ALP activity, the differentiated cells cultured for 21 days were fixed with $10 \%$ formalin and stained using the Gomori calcium-cobalt method. Briefly, the cells were washed with PBS for $5 \mathrm{~min}$. An incubation solution containing $5 \mathrm{ml}$ $2 \%$ barbital sodium, $5 \mathrm{ml} 3 \% \beta$-sodium glycerophosphate (Sigma Chemical Co.), $10 \mathrm{ml} 2 \%$ calcium nitrate, $5 \mathrm{ml} 2 \%$ magnesium sulfate and $25 \mathrm{ml}$ distilled water was placed on each slide followed by incubation for $4 \mathrm{~h}$ at $37^{\circ} \mathrm{C}$. The slides were then washed with distilled water and incubated in $2 \%$ calcium nitrate for $2 \mathrm{~min}$ at room temperature. Subsequently, the slides were incubated in $2 \%$ cobaltous nitrate for $2 \mathrm{~min}$ at room temperature. The slides were then washed with distilled water and incubated in $1 \%$ ammonium sulfide for $1 \mathrm{~min}$ at room temperature. The slides were then washed with running tap water and left to dry.

To detect extracellular matrix calcification with Alizarin red staining, on day 21 of differentiation, the osteogenic medium-cultured cells were fixed with $10 \%$ formalin. The cells were washed with PBS for $5 \mathrm{~min}$. A 2\% Alizarin red solution (Sigma Chemical Co.) was placed on each slide followed by incubation for $10 \mathrm{~min}$ at room temperature. The slides were then washed with running tap water for 5 min and left to dry.

Biometrics preparation of $n H A C / P L A$ grafts. The nHAC/PLA material (Beijing Allgens Medical Science and Technology Co., Ltd., China, http://www.allgensmed. com/cn/index.aspx) mimics the composition and the microstructure characteristics of the natural bone, the porosity is $80 \pm 10 \%$, the pore size is $300 \pm 250 \mu \mathrm{m}$ and the calcium phosphate content is $45 \pm 5 \%$. The mechanical strength is $\geq 0.8 \mathrm{Mpa}$. The nHAC/PLA materials were cut into $10 \times 4 \times 3$ or $5 \times 4 \times 3 \mathrm{~mm}$ blocks. The samples were then rinsed with $100 \%$ alcohol in order to remove organic residues and with double distilled water in order to remove inorganic residues (each solution for $10 \mathrm{~min}$ ). The samples were then sterilized by cobalt 60 .

Seeding of cells onto nHAC/PLA grafts. The PDLSCs isolated from the rats in the sham and OVX groups were respectively seeded onto the nHAC/PLA scaffold. The constructs were incubated in ODM for $2 \mathrm{~h}$ at $37^{\circ} \mathrm{C}$, allowing the cells to adhere to the scaffolds and $1 \mathrm{ml}$ of additional ODM was then added. The medium was replaced by ODM with or without E2 on day 4 of incubation and the grafts were then ready for in vitro experiments and in vivo implantation.

Scanning electron microscopy. The PDLSCs isolated from the rats in the sham and OVX group were respectively seeded onto the nHAC/PLA scaffolds at $1 \times 10^{6} \mathrm{cells} / \mathrm{cm}^{2}$ per graft, and were then cultured in ODM. Fixative was prepared from 2\% paraformaldehyde and 2.5\% glutaraldehyde (Sigma Chemical Co.) in $0.1 \mathrm{~mol} / \mathrm{l}$ phosphate buffer. Following fixation $\left(30 \mathrm{~min}, 37^{\circ} \mathrm{C}\right)$, the samples from $\mathrm{nHAC} / \mathrm{PLA}$, sham PDLSCs + nHAC/PLA, OVX PDLSCs + nHAC/PLA and 
OVX PDLSCs + nHAC/PLA + E2 cultured in ODM for 7 days were rinsed twice in PBS for $10 \mathrm{~min}$ and then washed 5 times (15 min each) in different ethanol concentrations (50, 75,90 and $95 \% \mathrm{v} / \mathrm{v}$ ethanol) in distilled water and 3 times for $10 \mathrm{~min}$ each in analytical ethanol. After the ethanol washes, the samples were rinsed in a series of different hexamethyldisilazane (HMDS) concentrations $(33.3,50$ and $66.6 \% \mathrm{v} / \mathrm{v})$ in analytic ethanol and 3 times in 100\% HMDS (1 min each). The morphological characterization of the cells and materials was carried out by means of scanning electron microscopy (SEM) using a Quanta 200 ESEM/SEM, FEI (Phillips, Madison, WI, USA) with beam energies of $6-25 \mathrm{kV}$ and fitted with an energy dispersive spectroscopy (EDS) apparatus. The samples were glued with conducing paste to appropriate mounting stabs, which were then coated with a several nanometer-thick layer of gold. The samples were examined under a Hitachi S-520 scanning electron microscope (Hitachi, Tokyo, Japan).

Estimation of ALP activity and osteocalcin (OCN) secretion. For the experiment, the cells were divided into 3 groups: sham PDLSCs + nHAC/PLA, OVX PDLSCs + nHAC/PLA and OVX PDLSCs + nHAC/PLA + E2. The cells at passage 4 were plated in 6-well plates, at $1 \times 10^{5}$ cells $/ \mathrm{ml}$, with DMEM containing $10 \%$ FBS for $12 \mathrm{~h}$ to allow attachment. The serum-containing medium was removed and replaced with a medium without serum for $12 \mathrm{~h}$, and the cells were then cultured in ODM. After 1-7, 7-14 and 14-21 days, the medium was collected from the wells.

ALP activity in the medium was assayed using a biochemistry automatic analyzer (Hitachi 7600; Hitachi).

OCN in the medium was assayed using a mouse-specific IRMA (Immutopics, Inc., San Clemente, CA, USA). Briefly, the sample containing mouse OCN was incubated stimultaneously with an antibody-coated bead and the 125I-labeled antibody. OCN contained in the sample is immunologically bound by the immobilized antibody and the radiolabeled antibody to form a 'sandwich' complex: bead/anti-mouse OCN, mouse OCN, 125I-anti-mouse OCN. At the end of the overnight incubation period, the bead was washed to remove any unbound labeled antibody and other components. The radioactivity bound to the bead was measured in a gamma counter. The radioactivity of the bound antibody complex is directly proportional to the bound antibody complex in the sample. As the amount of extracellular matrix proteins interferes with the total cellular protein determination, the data were determined and expressed (as $\mathrm{ng} / \mathrm{ml}$ ) for each culture dish.

Mineral formation assay. For this experiment, the cells were divided into 3 groups as follows: the sham PDLSCs $s+n H A C / P L A$, OVX PDLSCs + nHAC/PLA and OVX PDLSCs + nHAC/PLA + E2 groups. The PDLSCs were respectively seeded on $\mathrm{nHAC} / \mathrm{PLA}$ at $1 \times 10^{5} \mathrm{cells} / \mathrm{cm}^{2}$ per well/graft for $24 \mathrm{~h}$ to allow attachment in growth medium. The cells were starved using serum-free medium containing $2 \%$ BSA, $100 \mathrm{U} / \mathrm{ml}$ penicillan and $100 \mathrm{U} / \mathrm{ml}$ streptomycin for $24 \mathrm{~h}$. The cells were then cultured in ODM. After 21 days of culture, the samples were rinsed with PBS 3 times, fixed with $10 \%$ formalin, rinsed with PBS, and stained at room temperature for $20 \mathrm{~min}$ with Alizarin red solution. After staining, all constructs were washed with distilled water until the supernatant was clear.
Digital images were recorded. For optical density measurements, each construct was eluted for $30 \mathrm{~min}$ with $1 \mathrm{ml} 10 \%$ cetylpyridinium chloride monohydrate. The optical density at $540 \mathrm{~nm}$ was determined using a universal microplate reader (ELx800UV, Bio-Tek Instruments, Inc., Bad Friedrichshall, Germany). The optical density was calculated by taking the average optical absorbance of constructs grown in ODM.

Real-time-polymerase chain reaction (RT-PCR). For the experiment, the cells were divided into 3 groups as follows: the sham PDLSCs + nHAC/PLA, OVX PDLSCs + nHAC/PLA and OVX PDLSCs + nHAC/PLA + E2 groups. The PDLSCs were respectively seeded on $\mathrm{nHAC} / \mathrm{PLA}$ at $1 \times 10^{5}$ cells $/ \mathrm{cm}^{2}$ graft for $24 \mathrm{~h}$ to allow attachment in ordinary medium. The cells were starved using serum-free medium containing $2 \% \mathrm{BSA}, 100 \mathrm{U} / \mathrm{ml}$ penicillan and $100 \mathrm{U} / \mathrm{ml}$ streptomycin for $24 \mathrm{~h}$. The constructs were then cultured in ODM with or without $10^{-7} \mathrm{M} \mathrm{E} 2$. After 14 days of culture, the cells were crushed in lysis buffer (Roche, Indianapolis, IN, USA) with an RNase-free piston (Pellet), vortexed and spun. The clear cell lysate was transferred to QiaShredder (Qiagen, Inc., Valencia, CA, USA) columns for RNA purification. RNase-free DNase (Roche) was used to eliminate DNA contamination of RNA samples. Purified RNA was dissolved in RNase-free water and its concentration was assessed by reading at $260 \mathrm{~nm}$. RNA quality was checked on a $2 \%$ agarose gel with $1 \mathrm{mg} / \mathrm{ml}$ ethidium bromide. The samples were stored at $-80^{\circ} \mathrm{C}$ until use. Complementary DNA (cDNA) was synthesized from $2 \mu \mathrm{g}$ of total RNA using the First Strand cDNA synthesis kit for RT-PCR (AMV; Roche).

cDNA mixture $(8 \mu \mathrm{l})$ diluted 1:20 in water was subjected to real-time PCR using SYBR-Green I dye (LightCycler FastStart DNA master SYBR-Green I; Roche, Penzberg, Germany). Reactions were performed in $20 \mu \mathrm{l}$ PCR mixture containing $4 \mu 1$ 5X Master Mix (dNTP mixture with dUTP instead of dTTP, $\mathrm{MgCl}_{2}$, SYBR-Green I dye, TaqDNA polymerase and reaction buffer), $2 \mu 1$ of $10 \mu \mathrm{M}$ primers. The primer sequences (Shanghai Sangon Biological Engineering Technology and Services Co., Ltd., Shanghai, China) for ALP, OCN, ER $\alpha$, ER $\beta$ and $\beta$-actin are listed in Table I. $\beta$-actin real-time PCR was run as a control to monitor RNA integrity and to be used for normalization. The specificity of each primer pair was confirmed by melting curve analysis.

Histological and morphometric analysis of the in vivo experiments. For this experiment, the cells were divided into 4 groups as follows: the nHAC/PLA, sham PDLSCs + nHAC/PLA, OVX PDLSCs + nHAC/PLA and OVX PDLSCs + nHAC/PLA + E2 groups. The PDLSCs were seeded onto nHAC/PLA scaffolds at $1 \times 10^{8}$ cells $/ \mathrm{cm}^{2}$ per graft. Following 7 days of culture in vitro, the engineered constructs were then implanted into the backs of 8 severe combined immunodeficient (SCID) mice (age, 6-8 weeks; weight, $20 \mathrm{~g}$ ) for in vivo bone regeneration. Briefly, the SCID mice were anesthetized by an injection of pentobarbital sodium $(70 \mathrm{mg} / \mathrm{kg})$ to the abdominal cavity, and the skin on the backs of the mice was disinfected with iodine. After a small incision was made, the 4 grafts were transplanted into the backs of the SCID mice using tweezers, and the incision was then sutured. After 12 weeks, the SCID mice were sacrificed for removing the implanted constructs. The harvested constructs were then fixed 
Table I. Primers used for real-time PCR with SYBR-Green.

\begin{tabular}{|c|c|c|c|}
\hline Gene & Primer sequence & Species & $\begin{array}{l}\text { Product } \\
\text { size (bp) }\end{array}$ \\
\hline $\mathrm{ER} \alpha$ & $\begin{array}{l}\text { F: 5'-CAT CGA TAA GAA CCG GAG GA-3' } \\
\text { R: 5'-AAG GTT GGC AGC TCT CAT GT-3' }\end{array}$ & Rat & 190 \\
\hline $\mathrm{ER} \beta$ & $\begin{array}{l}\text { F: 5'-AGC AAC TGG TGC TCA CCC T-3' } \\
\text { R: 5'-GTC CGC CAG CTT AGT GAG G-3' }\end{array}$ & Rat & 94 \\
\hline ALP & $\begin{array}{l}\text { F: 5'-TCC CAC GTT TTC ACG TTT-3' } \\
\text { R: 5'-GAG ACG TTC TCC CGT TCA C-3' }\end{array}$ & Rat & 140 \\
\hline OCN & $\begin{array}{l}\text { F: 5'-TGA GGA CCC TCT CTC TGC TC-3 } \\
\text { R: 5'-AGG TAGCGC CGG AGT CTA TT-3 }\end{array}$ & Rat & 150 \\
\hline$\beta$-actin & $\begin{array}{l}\text { F: 5'-CCC ATC TAT GAG GGT TAC GC-3' } \\
\text { R: 5'-TTT AAT GTC ACG CAC GAT TTC-3' }\end{array}$ & Rat & 150 \\
\hline
\end{tabular}

F, forward; R, reverse; ER, estrogen receptor; OCN, osteocalcin.

in $10 \%$ formalin neutral buffer solution at $\mathrm{pH} 7.4$ for 2 days and were then trimmed using waterproof polishing paper without demineralization and cut into $5-\mu \mathrm{m}$-thick sections, and stained with hematoxylin and eosin (H\&E) for light microscopic observation. All the samples were analyzed microscopically and compared with the controls. Digital images were recorded.

For morphometric analysis, 5 sequential sections per implant were selected for evaluation under low magnification, allowing the coverage of the entire implant. Using a Leica-Qwin 3.2 image analysis system (Leitz DMRD; Leica Microsystems Inc., Bannockburn, IL, USA), all slides were analyzed to identify the type of tissue (mature bone-like and osteoid-like). The extent of newly formed mature bone and osteoid was indicated by the percentage of bone formation area within the section, Total scores per section were calculated and averaged for all sections to obtain an overall score for each implant.

Statistical analysis. All data are presented as the means \pm standard deviation (SD). The statistical analyses of the results were performed using computer-based SPSS 16.0 software. A one-way analysis of variance with the post hoc test LSD for multiple comparisons was used to determine statistically significant differences in the cell proliferation, mineral formation, the expression levels of ALP, OCN, ER $\alpha$ and ER $\beta$ mRNAs and the percentage of bone formation area between the study groups. The repeated measures were used to determine the statistically significant differences in ALP activity and OCN secretion. Values of $\mathrm{P}<0.05$ were considered to indicate statistically significant differences.

\section{Results}

Effects of ovariectomy on body weight and BMD, and estrogen levels in rats. There were no significant differences in body weight, BMD and estrogen levels between the rats in the sham and OVX groups before the experiment. Three months after the ovariectomy, the body weight of the rats in the OVX group was significantly higher than that of the rats in the sham group; however, the BMD and estrogen levels of the rats in the OVX group were significantly lower than those of the rats in the sham group (Fig. 1).

Morphology and identification of rat PDLSCs. Morphologically, the PDLSCs isolated from the rats in the sham group (Fig. 2A) and OVX group (Fig. 2B) had a triangular, spindle and fusiform shape, and expressed STRO-1, as shown by immunohistochemical staining (Fig. 2C and D). They also expressed vimentin (Fig. 2E and G), but did not express keratin (Fig. 2F and $\mathrm{H}$ ), as shown by immunofluorescence staining. Vimentin was labeled red with TRITC, and the nucleus was labeled blue with DAPI.

Effect of estrogen on the proliferation of PDLSCs isolated from $O V X$ rats. To observe the differences in the proliferation of cells from the sham and OVX group, and to examine the effects of estrogen on the proliferation of cells from the OVX group, MTT assay was carried out to monitor the proliferation of the 3 groups of cells (sham group, OVX group and OVX + group). The results revealed that the proliferation of the cells in the 3 groups increased gradually with the extension of the culture time, and that the proliferation of the cells in the OVX and OVX + E2 groups reached peak levels on on day 7 . Following culture for 2, 3, 4, 5, 6 and 7 days, the proliferation of the cells in the OVX group was significantly greater than that of the cells in the sham group. The proliferation of the cells in the OVX + E2 group was significantly lower than that of the cells in the OVX group following culture for 2, 3, 4, 5, 6, 7 and 8 days, and was significantly greater than that of the cells in the sham group following culture for 2, 3, 4, 5 and 6 days (Fig. 3).

Effects of estrogen on the osteogenic differentiation of the PDLSCs isolated from OVX rats. Following culture in ODM for 21 days, the cells from the sham group exhitibed intense staining for ALP (Fig. 4A), and also exhibited calcium deposition, with formed and developed mineralization nodules (Fig. 4D), as revealed by the Gomori calcium-cobalt method and Alizarin red staining. Weaker staining was observed in the cells from the OVX group (Fig. 4B and E). 


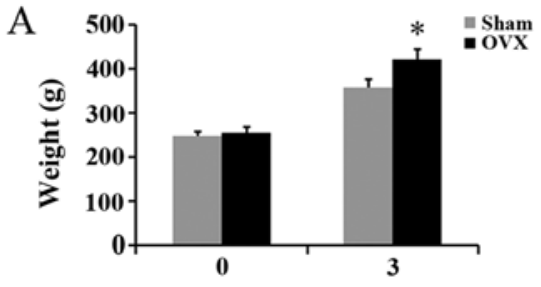

Time (months)
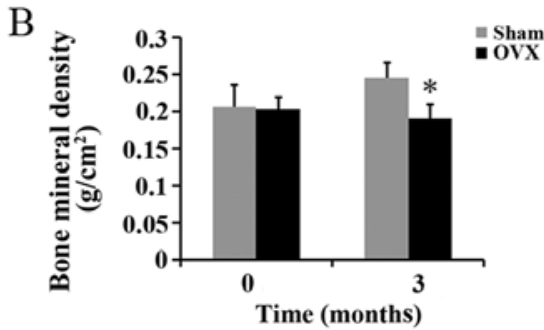

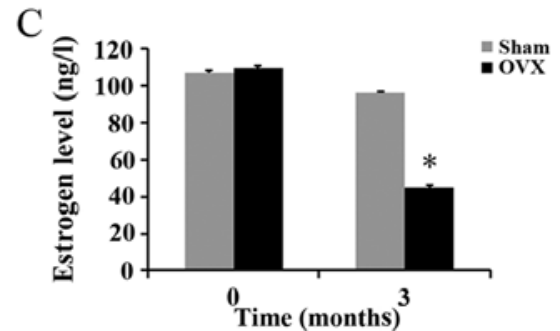

$\mathrm{C}$

Time (months)

Figure 1. Effects of ovariectomy at 3 months after surgery on (A) body weight, (B) bone mineral density (BMD) and (C) estrogen level in the sham-operated (sham) and ovariectomized (OVX) group rats ( $\mathrm{n}=24$ /group, means $\pm \mathrm{SD}$ ). ${ }^{*} \mathrm{P}<0.05$, compared to sham group at the same time point.
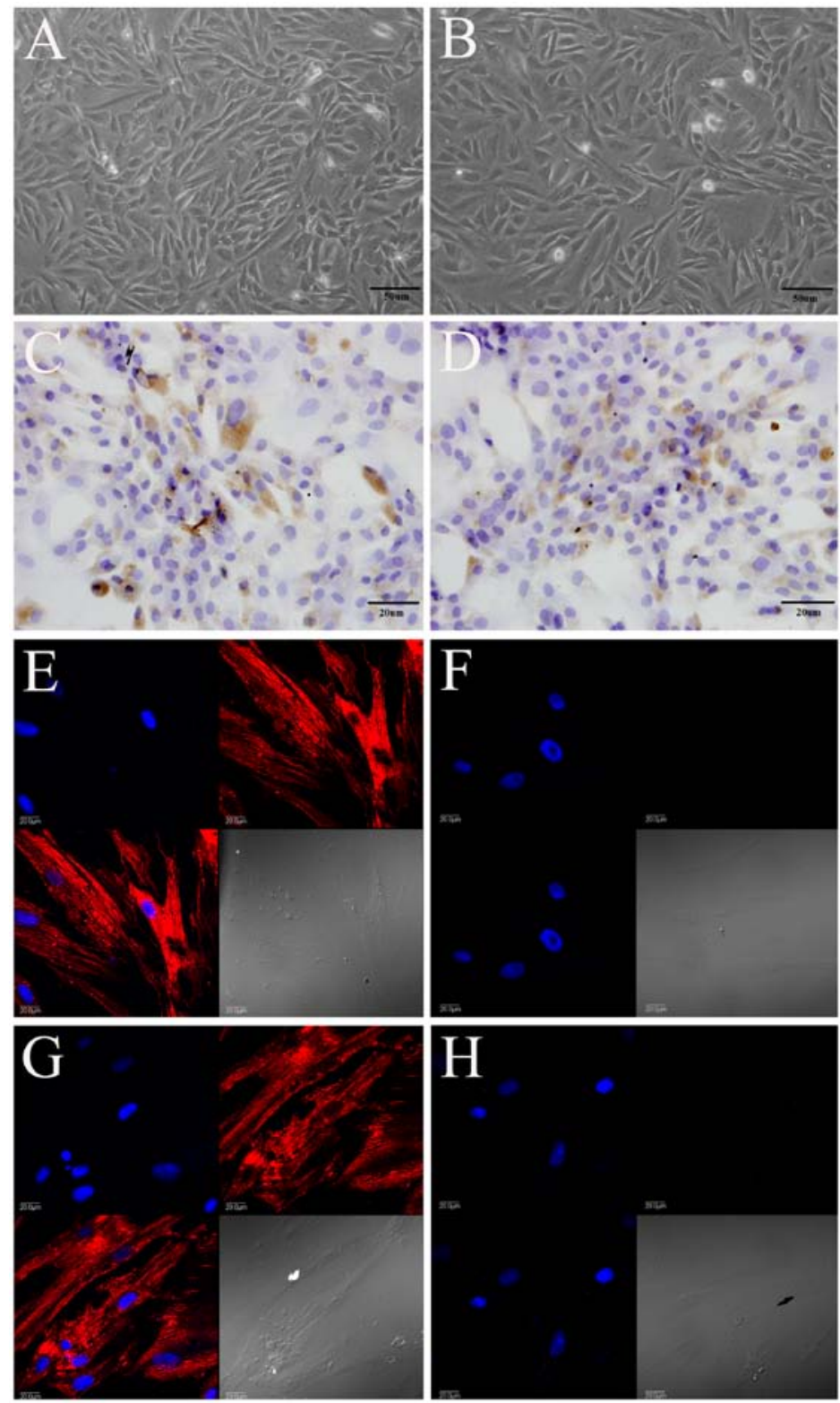

Figure 2. The periodontal ligament stem cells (PDLSCs) isolated from rats in the (A, C, E and G) sham-operated (sham) group and (B, D, F and H) ovariectomized (OVX) group expressed (C and D) STRO-1 and (E and G) vimentin, but did not express (F and H) keratin. (A and B) Magnification, x200; (C and D) magnification, $\mathrm{x} 400$; (E-H) magnification, $\mathrm{x} 600$.

When the cells from the OVX group were cultured in $\mathrm{ODM}+10^{-7} \mathrm{E} 2$ for 21 days, the cells exhibited intense staining for ALP (Fig. 4C), and also exhibited calcium deposition, with formed and developed mineralization nodules (Fig. 4E).
Scanning electron microscopy. The results of SEM revealed that the nHAC/PLA had the micro-structure characteristics of natural bone (Fig. 5A). When the constructs were cultured in ODM for 7 days, a large number of cells in the sham 


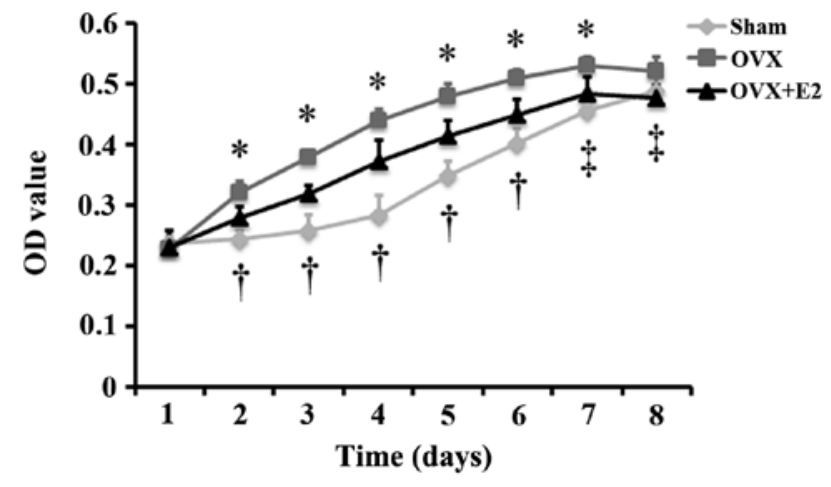

Figure 3. Effect of estrogen on the proliferation of periodontal ligament stem cells (PDLSCs) isolated from rats in the ovariectomized (OVX) group over a period of 8 days $(n=12$ wells/plate, means $\pm S D)$. At the same time-point, ${ }^{*} \mathrm{P}<0.05, \mathrm{OVX}$ vs. sham or OVX $+\mathrm{E} 2$; ${ }^{\dagger} \mathrm{P}<0.05, \mathrm{OVX}+\mathrm{E} 2$ vs. sham; ${ }^{+} \mathrm{P}<0.05$, OVX + E2 vs. OVX. Sham, sham-operated group.

PDLSCs + nHAC/PLA (Fig. 5B), OVX PDLSCs + nHAC/PLA (Fig. 5C) and OVX PDLSCs + nHAC/PLA + E2 (Fig. 5D) groups had adhered to, and had expanded and proliferated on the nHAC/PLA. There were many filamentous extracellular matrices on the surface of the cells. Some of the cells in the sham PDLSCs + nHAC/PLA and OVX PDLSCs + nHAC/PLA + E2 groups were covered by more mineral deposits.

Effects of estrogen on ALP activity and OCN secretion in the PDLSCs isolated from OVX rats and seeded on $n H A C / P L A$. The specific ALP activity of the PDLSCs from the 3 groups reached the highest levels during the culture period of 7-14 days. Compared with the cells isolated from the sham-operated rats, the ovariectomy significantly decreased ALP activity in the cells during the culture period of 1-7, 7-14 and 14-21 days. Nevertheless, treatment with E2 significantly increased ALP activity in the cells isolated from the OVX rats, and the value of ALP activity in the OVX PDLSCs + nHAC/PLA + E2 group was significantly higher than that of the sham PDLSCs + nHAC/PLA group during the culture period 7-14 days (Fig. 6A).

OCN secretion from the PDLSCs in the 3 groups gradually increased with the extension of the culture time. Compared with the cells isolated from the sham-operated rats, the ovariectomy significantly decreased OCN secretion from the cells isolated from the OVX rats during the culture period of 1-7, 7-14 and 14-21 days. Nevertheless, E2 significantly increased OCN secretion from the cells isolated from the OVX rats, and the value of OCN secretion in the OVX PDLSCs + nHAC/PLA + E2 group was significantly higher than that of the sham PDLSCs + nHAC/PLA group during the culture period of 14-21 days (Fig. 6B).

Effect of estrogen on mineral formation in the PDLSCs isolated from OVX rats and seeded on the nHAC/PLA scaffold. Alizarin red staining was used to quantify calcium phosphate mineral formation in the cells on the sham PDLSCs + nHAC/PLA, OVX PDLSCs + nHAC/PLA and OVX PDLSCs + nHAC/PLA + E2 constructs following culture for 21 days. The mineral formation in the cells on the OVX PDLSCs + nHAC/PLA construct was significantly less than that of the cells on the sham PDLSCs + nHAC/PLA and OVX PDLSCs + nHAC/PLA + E2 (Fig. 9A-C) constructs, and mineral formation in the cells on the OVX PDLSCs + nHAC/PLA + E2 construct was significantly greater than that of the cells on the sham PDLSCs + nHAC/PLA (Fig. 7A).

Effect of estrogen on the mRNA expression levels of ALP, $O C N, E R \alpha$ and $E R \beta$ in the PDLSCs isolated from OVX rats and seeded on $n H A C / P L A$. When the PDLSCs from the shamoperated and OVX rats were seeded on the nHAC/PLA and were cultured in ODM with or without E2 for 14 days, realtime PCR analysis revealed that the mRNA expression levels of ALP (Fig. 7B), OCN (Fig. 7C), ER $\alpha$ (Fig. 7D) and ER $\beta$ (Fig. 7E) in the PDLSCs from the OVX rats were significantly downregulated due to the ovariectomy. E2 significantly increased the mRNA expression levels of ALP, OCN, ER $\alpha$ and ER $\beta$ in
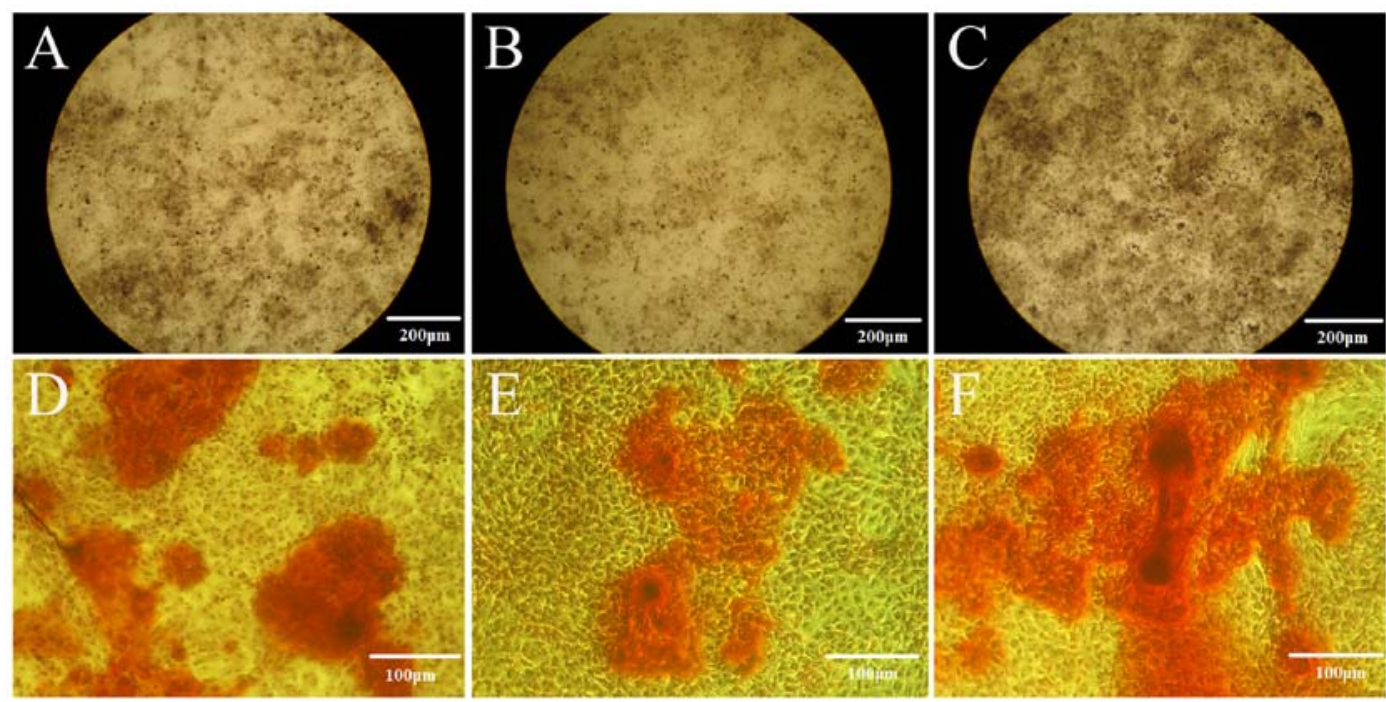

Figure 4. Effect of estrogen on the osteogenic differentiation of periodontal ligament stem cells (PDLSCs). The cells from the (A and D) sham group, (B and E) OVX group and (C and F) OVX + E2 group were stained for (A-C) ALP and (D-F) mineralization. (A-C) Magnification, $\mathrm{X}$ 40; (D-F) magnification, x100. OVX, ovariectomized; sham, sham-operated. 


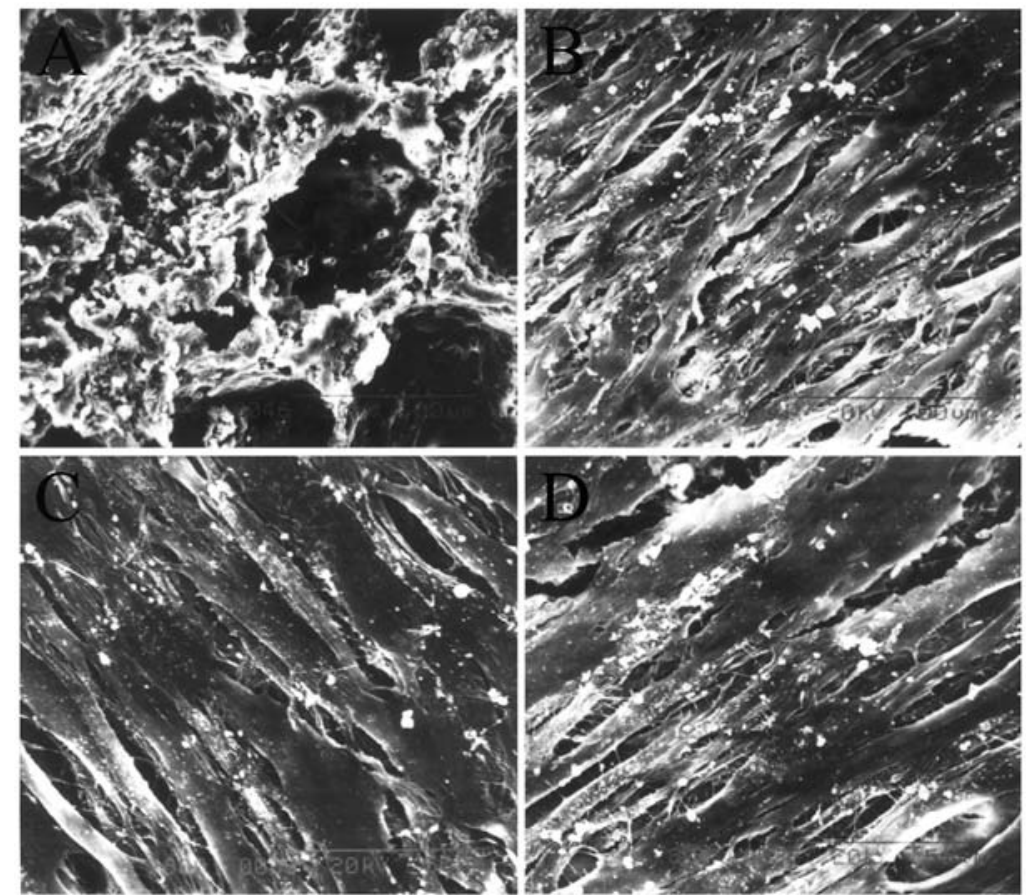

Figure 5. Results from SEM showing the structure of (A) nHAC/PLA and the morphology of the cells in the (B) sham PDLSCs + nHAC/PLA, (C) OVX PDLSCs + nHAC/PLA and (D) OVX PDLSCs + nHAC/PLA + E2. (A) Magnification, x400; (B-D) magnification, x800. OVX, ovariectomized; PDLSCs, periodontal ligament stem cells; nHAC/PLA, nano-hydroxyapatite/collagen/poly(L-lactide).
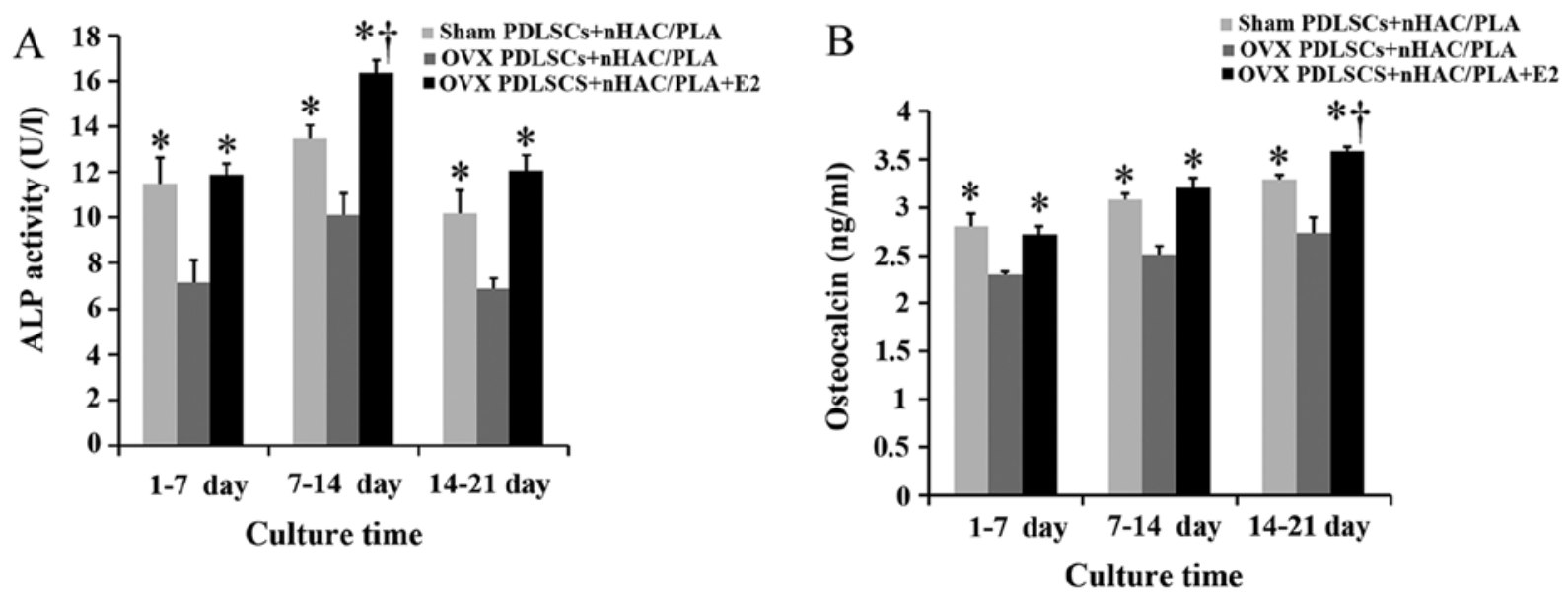

Figure 6. Effect of estrogen on (A) ALP activity and (B) osteocalcin secretion in OVX PDLSCs + nHAC/PLA (n=8 wells/plate, means \pm SD). At same time period, "P<0.05, compared to OVX PDLSCs + nHAC/PLA; ${ }^{\mathrm{P}}<0.05$, compared to sham PDLSCs + nHAC/PLA. OVX, ovariectomized; PDLSCs, periodontal ligament stem cells; nHAC/PLA, nano-hydroxyapatite/collagen/poly(L-lactide).

the PDLSCs from the OVX rats, and the expression levels in the OVX PDLSCs + nHAC/PLA + E2 group were significantly higher than those in the sham PDLSCs + nHAC/PLA group.

Histological and morphometric analysis of in vivo experiments. The PDLSCs isolated from the sham-operated and OVX rats were seeded onto the nHAC/PLA porous scaffolds and cultured in ODM or ODM + E2 for 7 days, and the cell/scaffold constructs were then implanted subcutaneously into SCID mice. After 12 weeks, the subcutaneous implants were removed from the mice and analyzed for new bone formation. H\&E staining revealed that nHAC/PLA alone exhibited no new bone formation, while the marked ingrowth of soft connective tissue into the grafts was observed (Fig. 8A). Newly formed bones were observed in the sham PDLSCs + nHAC/PLA (Fig. 8B), OVX PDLSCs + nHAC/PLA (Fig. 8C) and OVX PDLSCs + nHAC/PLA + E2 (Fig. 8D) constructs. Newly formed blood vessels were observed in the micropore of the nHAC/PLA. Osteoblastic cells lined the surface of the newly formed bone.

The results of histomorphometric analysis are summarized in Fig. 9. After 12 weeks of implantation, the percentages of bone formation area between the groups differed significantly. The percentage of bone formation area in the sham PDLSCs + nHAC/PLA group $(5.498 \pm 0.65 \%)$ and was significantly higher than that of the OVX PDLSCs + nHAC/PLA group $(3.269 \pm 0.72 \%)$, but was significantly lower than that of 


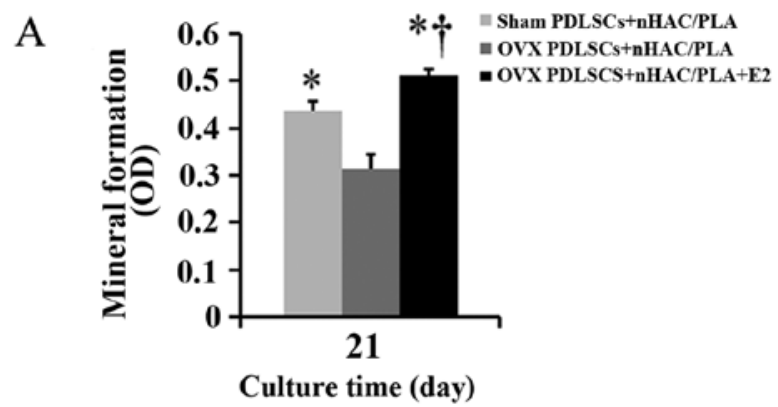

B

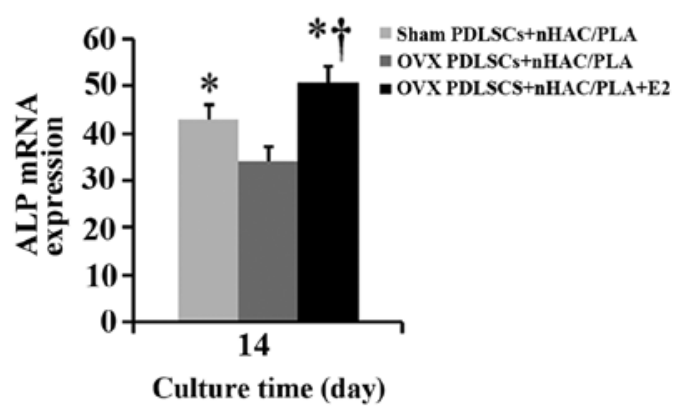

$\mathrm{D}$

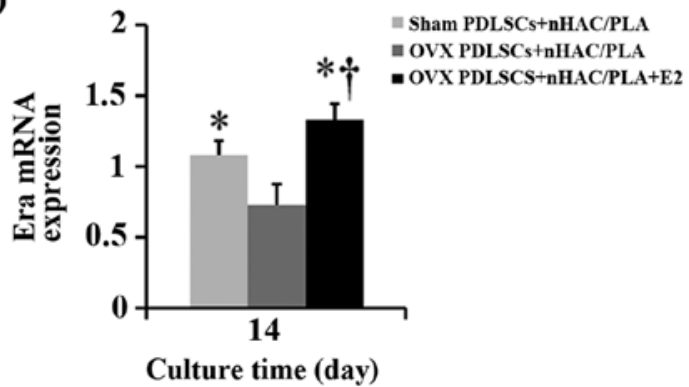

$\mathrm{C}$

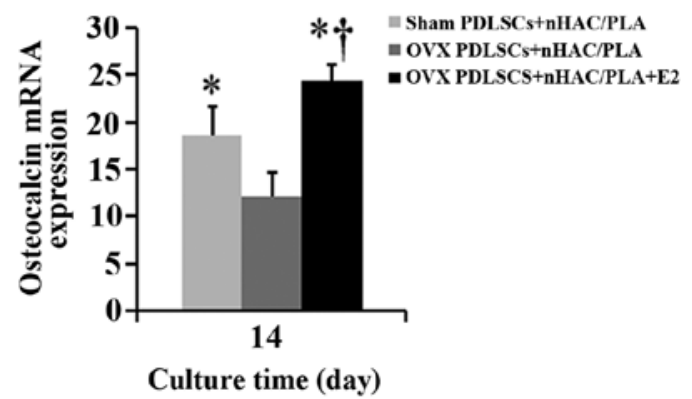

E

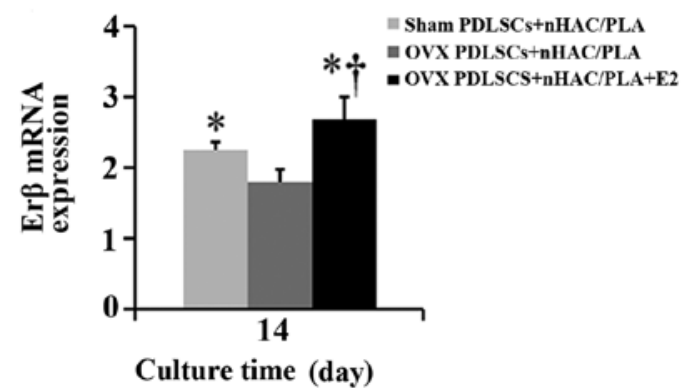

Figure 7. Effect of estrogen on (A) mineral formation and (B) ALP, (C) OCN, (D) ER $\alpha$ and (E) ER $\beta$ mRNA expression in OVX PDLSCs + nHAC/PLA $(\mathrm{n}=6$ wells/plate, means $\pm \mathrm{SD}) .{ }^{*} \mathrm{P}<0.05$, compared to OVX PDLSCs $+\mathrm{nHAC} / \mathrm{PLA} ;{ }^{\dagger} \mathrm{P}<0.05$, compared to sham PDLSCs + nHAC/PLA. OVX, ovariectomized; PDLSCs, periodontal ligament stem cells; nHAC/PLA, nano-hydroxyapatite/collagen/poly(L-lactide).

the OVX PDLSCs + nHAC/PLA + E2 group (7.573 $\pm 1.1 \%)$. The nHAC/PLA alone exhibited no bone formation.

\section{Discussion}

Autologous adult stem cell-based tissue engineering and regenerative medicine has been considered a promising substitute for current clinical treatments that restore tissue and organ deficiencies (39-41). Successful tissue regeneration requires a sufficient cell population with high differentiation potential.

PDLSCs can regenerate new functional periodontal support tissue, including cementum, alveolar bone and periodontal ligament fibers. Therefore, PDLSC-based tissue engineering has now emerged as a promising and ideal alternative approach for the clinical treatment of periodontal tissue defects $(22,23)$. However, a number of factors (including advanced age, degenerative diseases of donors and the microenvironment of systemic disease, such as hyperglycemia and estrogen deficiency) affect the PDLSC population, the proliferation rate and the differentiation potential $(32,37,42)$, indicating that the changes in the microenvironment are responsible for PDLSCs exhibiting different characteristics.
In light of the above findings, the aim of the present study was the detailed characterization of the properties of PDLSCs derived from OVX rats. An ovariectomy was performed in order to create an estrogen-deficient microenvironment. A number of studies have suggested that an ovariectomy can create an estrogen-deficient microenvironment (32,43-46). In this study, the weight, BMD of the lumbar spine and estrogen levels in the 2 groups of rats (sham-operated and OVX rats) were examined, an these values differed significantly between the sham and OVX group at the 3rd month after surgery, but not at day 0 after surgery. The OVX rats had a higher weight, and lower BMD and a lower estrogen level. These results demonstrated that we successfully created an estrogen-deficient microenvironment by ovariectomy.

Our first goal was to define the proliferative ability of the PDLSCs in regards estrogen depletion. In our primary PDLSC cultures, we observed that morphologically, the 2 groups of cells had a triangular, spindle and fusiform shape, with no significant differences between the 2 groups. The PDLSCs from the sham group attached more easily, and expanded earlier than those from the OVX group. After the primary cells were passaged, the PDLSCs from the OVX group proliferated more rapidly. This 

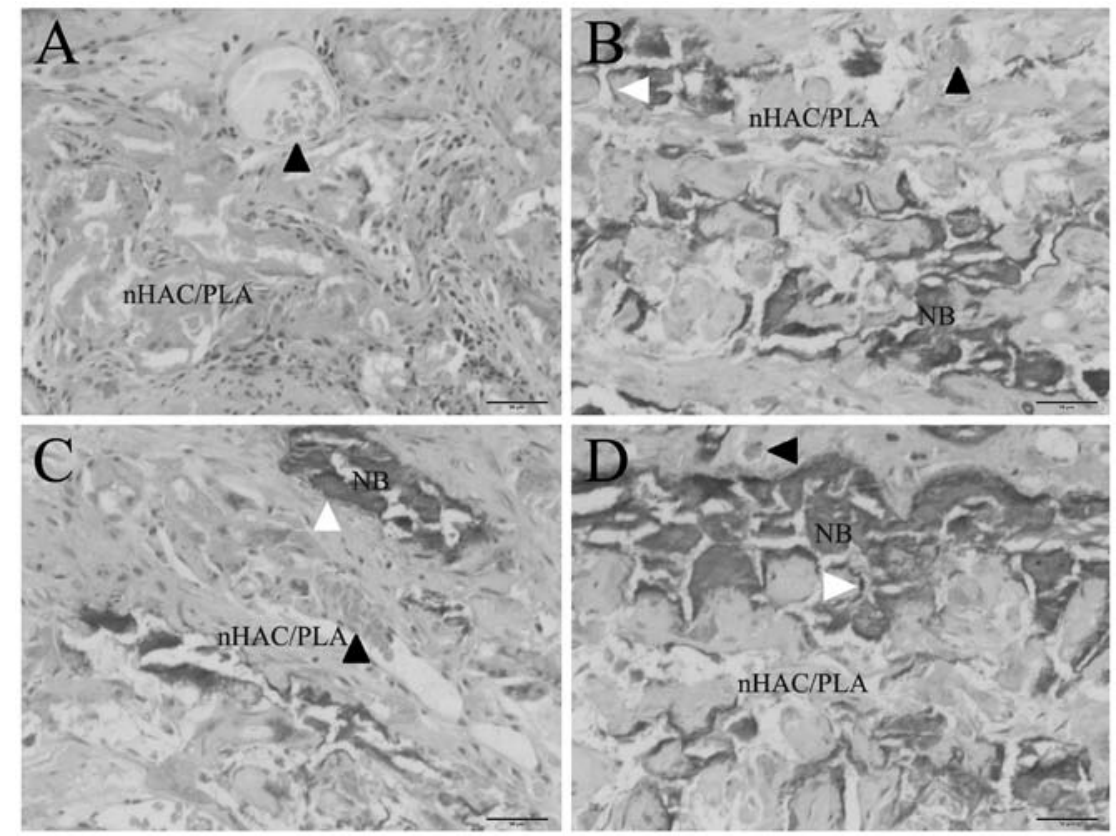

Figure 8. In vivo osteogenesis. (A) nHAC/PLA, (B) sham PDLSCs + nHAC/PLA, (C) OVX PDLSCs + nHAC/PLA, (D) OVX PDLSCs + nHAC/PLA + E2 Magnification, x200, NB, newly formed bone. Black arrowheads indicate blood vessels, white arrowheads indicate osteoblastic cells. OVX, ovariectomized PDLSCs, periodontal ligament stem cells; nHAC/PLA, nano-hydroxyapatite/collagen/poly(L-lactide).

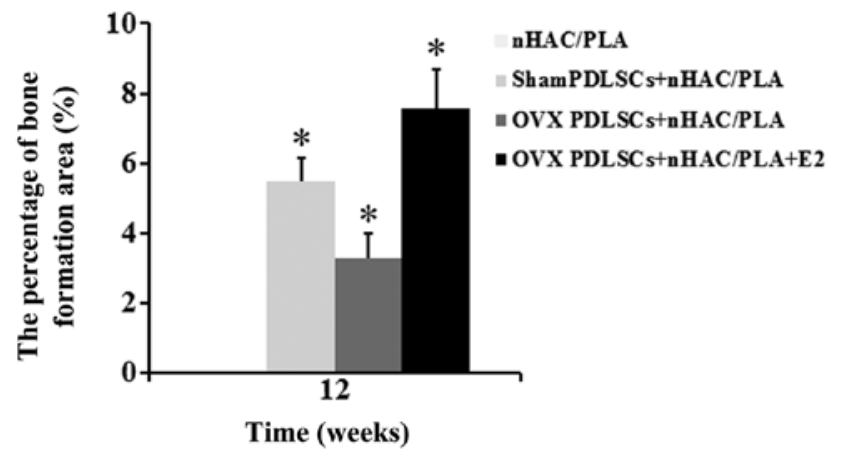

Figure 9. Percentage of bone formation area in each group after 12 weeks of implantation ( $\mathrm{n}=6$ constructs/group, mean $\pm \mathrm{SD}$ ). After 12 weeks, there were significant differences between all groups. Significance of the data at ${ }^{*} \mathrm{P}<0.05$.

indicated that the estrogen deficiency enhanced the proliferative ability of the PDLSCs. However, ALP staining and Alizarin red staining demonstrated that estrogen deficiency inhibited the osteogenic differentiation of the PDLSCs in monolayer culture conditions. These results were consistent with those reported in the study by Zhang et al (32). A possible explanation for the increased proliferation is that estrogen deficiency makes an organism produce more PDLSCs in a compensative manner. However, the increased proliferative ability of the PDLSCs failed to enhance the osteogenic differentiation ability, which is why postmenopausal women often suffer from osteoporosis. However, estrogen supplements can reverse the effects of estrogen deficiency. This may be why postmenopausal women adopt estrogen replacement therapy to reduce immediate and long-term excessive bone mass loss caused by low levels of estrogen, and to prevent or delay the onset of osteoporosis.

Our second goal was to analyze the osteogenic differentiation ability of PDLSCs derived from OVX rats cultured in $3 \mathrm{D}$ nHAC/PLA nanostructured scaffolds. A number of studies have demonstrated that the population, proliferation rate and differentiation potential of adult stem cells are affected by systemic diseases, lifestyle, drug consumption and aging $(24,26,32,37,42)$. The results from the study by Bressan et al (47) demonstrated that dental pulp stem cells derived from an aged group exhibited a low proliferative and osteogenic differentiation ability in monolayer culture conditions; when cultured on hydroxyapatite (HA) nanostructured granules and used in vivo to repair critical size defects, they exhibited the same ability as those of the younger group in terms of time to repair and the quality of extracellular matrix. Their study indicates that 3D HA nanostructured granules can reverse the effects of advanced age on the proliferative ability and the osteogenic differentiation ability of dental pulp stem cells. However, in the present study, the 3D nHAC/PLA nanostructured scaffolds did not alter the effects of estrogen deficiency on the osteogenic differentiation ability of the PDLSCs. When the PDLSCs were cultured on nHAC/PLA in ODM, the 3 groups of cells (sham PDLSCs + nHAC/PLA, OVX PDLSCs + nHAC/ PLA and OVX PDLSCs + nHAC/PLA + E2) all adhered, expanded and proliferated, and produced a large amount of mineral matrix, as shown by SEM; however, the ALP activity and OCN secretion in the cells derived from the OVX group decreased due to estrogen deficiency. The results from real-time PCR further confirmed this by assessing the intrinsic expression of osteogenic markers. In order to further examine the effects of estrogen deficiency on the osteogenic differentiation of PDLSCs derived from OVX rats, we also examined the mineral formation of the cells cultured in monolayer conditions and on the nHAC/PLA. The results revealed that, in monolayer conditions, more positive strong Alizarin red staining existed in the cells derived from the sham group. In 3D nHAC/PLA conditions, quantitative analysis of Alizarin red staining suggested that 
estrogen deficiency significantly decreased mineral formation in the cells. Previous studies have demonstrated that estrogen plays an important role in the osteogenic differentiation of PDLSCs by binding specific intracellular ER (29-32). Thus, we also examined the mRNA expression of ER in the cells derived from OVX rats. Estrogen deficiency downregulated the mRNA expression of ER $\alpha$ and ER $\beta$. There were no differences between the cells cultured on 3D nHAC/PLA nanostructured scaffolds in our study and the cells were cultured in monolayer conditions in a previous study (32). When the PDLSCs derived from OVX rats were treated with estrogen, the results revealed that the estrogen-treated PDLSCs exhibited increased ALP activity, OCN secretion and mineral formation, as well as an increased mRNA expression of ALP and OCN, and ER $\alpha$ and ER $\beta$. These results indicated that the estrogen-deficient microenvironment impaired the osteogenic differentiation of PDLSCs derived from OVX rats. Estrogen enhanced the osteogenic differentiation of PDLSCs derived from OVX rats and seeded on nHAC/PLA in vitro. Both $\mathrm{ER} \alpha$ and $\operatorname{Er} \beta$ were involved in the osteogenic differentiation of the PDLSCs.

In in vitro experiments, we demonstrated that treatment with estrogen restored the osteogenic differentiation capacity of the PDLSCs seeded on nHAC/PLA, which had been impaired due to estrogen deficiency. As a final goal, we examined whether the PDLSCs derived from OVX rats and seeded on 3D nanostructured scaffolds were able to be undergo osteogenesis in vivo. The cells seeded on the nHAC/PLA were cultured for 7 days in vitro, and the constructs were then implanted subcutaneously into the backs of SCID mice for 12 weeks, and we then observed that the impaired PDLSCs (OVX PDLSCs + nHAC/PLA) exhibited new bone formation abilities, but their regenerative ability was significantly lower than that of the other 2 groups (sham PDLSCs + nHAC/PLA and OVX PDLSCs + nHAC/PLA + E2). This ability was comparable between the estrogen-treated group and sham group.

In conclusion, to date, a number of studies have demonstrated that postnatal stem cells can be isolated from various adult tissues, such as bone marrow (25), skeletal muscle (48), brain (49), liver (50), pancreas (51), lungs (52), heart (53) and kidneys (54), and that these cells exhibit self-renewal capacity and multilineage differentiation potential. However, there are some disadvantages associated with harvesting stem cells, such as additional damage to the body. The harvesting of stem cells from various tissues or organs can damage those organs, such as in stem cells derived from bone marrow (25), skeletal muscle (48), brain (49), liver (50), pancreas (51), lungs (52), heart (53) and the kidneys (54). Therefore, we focused on utilizing tissues that can be obtained without additional injury, as well as on new stem cell sources, such as periodontal ligament stem cells from teeth extracted for orthodontic purposes, prophylactically extracted nondecayed third molar teeth. Periodontal ligament stem cells have many advantages, and the results to date suggest that teeth are a viable source of adult MSCs for a wide range of clinical applications $(22,23,37)$. Thus far, no investigation on the stemness of PDLSCs cultured on 3D nHAC/PLA nanostructured scaffolds and its correlation with estrogen deficiency and estrogen supplements has been performed, at least to the best of our knowledge.

Our data provide three important insights. The first is that this study clearly demonstrates that PDLSCs exhibit a response to estrogen deficiency at the 3rd month following an ovariectomy. The PDLSCs derived from OVX rats exhibit an increased proliferative ability and a weaker osteogenic differential ability. The second important conclusion is that if cells from OVX rats are cultured on 3D nHAC/PLA nanostructured scaffolds, they regain the same biological properties as those of cells cultured in monolayer conditions. Both $\operatorname{ER} \alpha$ and $\operatorname{Er} \beta$ are involved in the osteogenic differentiation of PDLSCs. The third conclusion is that estrogen can enhance the bone regeneration potential of periodontal ligament stem cells derived from OVX rats and seeded on nHAC/PLA in vivo. The impaired PDLSCs from OVX rats can form new bone, but their regenerative ability is limited. Further studies are required to investigate the in situ bone regenerative capacity of estrogen-treated PDLSCs derived from OVX rats and seeded on 3D nHAC/PLA nanostructured scaffolds.

\section{Acknowledgements}

The authors would like to thank the staff and faculty members of the Institute of Stomatology of the General Hospital of People's Liberation Army of China. This study was funded by the National High-tech R\&D Program (863 Program), grant no. 2013AA032201, the PLA General Hospital Clinical Research Support Fund, grant no. 2015FC-CXYY-1003.

\section{References}

1. Bertonazzi A, Nelson B, Salvador J and Umland E: The smallest available estradiol transdermal patch: a new treatment option for the prevention of postmenopausal osteoporosis. Womens Health (Lond Engl) 11: 815-824, 2015.

2. Daniell HW: Postmenopausal tooth loss. Contributions to edentulism by osteoporosis and cigarette smoking. Arch Intern Med 143: 1678-1682, 1983.

3. Tominari T, Hirata $\mathrm{M}$, Matsumoto $\mathrm{C}$, Inada $\mathrm{M}$ and Miyaura $\mathrm{C}$ : Polymethoxy flavonoids, nobiletin and tangeretin, prevent lipopolysaccharide-induced inflammatory bone loss in an experimental model for periodontitis. J Pharmacol Sci 119: 390-394, 2012.

4. Kobayashi M, Matsumoto C, Hirata M, Tominari T, Inada M and Miyaura C: The correlation between postmenopausal osteoporosis and inflammatory periodontitis regarding bone loss in experimental models. Exp Anim 61: 183-187, 2012.

5. Marques MR, da Silva MA, Manzi FR, Cesar-Neto JB Nociti FH Jr and Barros SP: Effect of intermittent PTH administration in the periodontitis-associated bone loss in ovariectomized rats. Arch Oral Biol 50: 421-429, 2005.

6. Duarte PM, de Assis DR, Casati MZ, Sallum AW, Sallum EA and Nociti FH Jr: Alendronate may protect against increased periodontitis-related bone loss in estrogen-deficient rats. J Periodontol 75: 1196-1202, 2004.

7. Reinhardt RA, Payne JB, Maze CA, Patil KD, Gallagher SJ and Mattson JS: Influence of estrogen and osteopenia/osteoporosis on clinical periodontitis in postmenopausal women. J Periodontol 70: 823-828, 1999.

8. Palomo L, Chitguppi R, Buencamino MC, Santos D and Thacker H: A need to educate postmenopausal women of their periodontal health. J Indian Soc Periodontol 17: 225-227, 2013.

9. Pepelassi E, Nicopoulou-Karayianni K, Archontopoulou AD Mitsea A, Kavadella A, Tsiklakis K, Vrotsos I, Devlin H and Horner K: The relationship between osteoporosis and periodontitis in women aged 45-70 years. Oral Dis 18: 353-359, 2012.

10. Haas AN, Rösing CK, Oppermann RV, Albandar JM and Susin C: Association among menopause, hormone replacement therapy, and periodontal attachment loss in southern Brazilian women. J Periodontol 80: 1380-1387, 2009.

11. Lerner UH: Inflammation-induced bone remodeling in periodontal disease and the influence of post-menopausal osteoporosis. J Dent Res 85: 596-607, 2006.

12. Taguchi A, Tanimoto K, Suei Y, Otani K and Wada T: Oral signs as indicators of possible osteoporosis in elderly women. Oral Surg Oral Med Oral Pathol Oral Radiol Endod 80: 612-616, 1995. 
13. Klemetti E: A review of residual ridge resorption and bone density. J Prosthet Dent 75: 512-514, 1996.

14. Hirai T, Ishijima T, Hashikawa Y and Yajima T: Osteoporosis and reduction of residual ridge in edentulous patients. J Prosthet Dent 69: 49-56, 1993.

15. von Wowern N and Kollerup G: Symptomatic osteoporosis: A risk factor for residual ridge reduction of the jaws. J Prosthet Dent 67: 656-660, 1992.

16. Gonçalves PF, Gurgel BC, Pimentel SP, Sallum EA, Sallum AW, Casati MZ and Nociti FH Jr: Effect of two different approaches for root decontamination on new cementum formation following guided tissue regeneration: A histomorphometric study in dogs. J Periodontal Res 41: 535-540, 2006.

17. Hoffmann T, Richter S, Meyle J, Gonzales JR, Heinz B, Arjomand M, Sculean A, Reich E, Jepsen K, Jepsen S and Boedeker RH: A randomized clinical multicentre trial comparing enamel matrix derivative and membrane treatment of buccal class II furcation involvement in mandibular molars. Part III: Patient factors and treatment outcome. J Clin Periodontol 33: 575-583, 2006

18. Needleman IG, Worthington HV, Giedrys-Leeper E and Tucker RJ: Guided tissue regeneration for periodontal infra-bony defects. Cochrane Database Syst Rev: Apr 19, 2006 (Epub ahead of print). doi: CD001724, 10.1002/14651858.CD001724.pub2.

19. Venezia E, Goldstein M, Boyan BD and Schwartz Z: The use of enamel matrix derivative in the treatment of periodontal defects: A literature review and meta-analysis. Crit Rev Oral Biol Med 15: 382-402, 2004.

20. Kaigler D, Cirelli JA and Giannobile WV: Growth factor delivery for oral and periodontal tissue engineering. Expert Opin Drug Deliv 3: 647-662, 2006.

21. Blumenthal NM: A clinical comparison of collagen membranes with e-PTFE membranes in the treatment of human mandibula buccal class II furcation defects. J Periodontol 64: 925-933, 1993.

22. Liu Y, Zheng Y, Ding G, Fang D, Zhang C, Bartold PM, Gronthos S, Shi S and Wang S: Periodontal ligament stem cellmediated treatment for periodontitis in miniature swine. Stem Cells 26: 1065-1073, 2008.

23. Seo BM, Miura M, Gronthos S, Bartold PM, Batouli S, Brahim J, Young M, Robey PG, Wang CY and Shi S: Investigation of multipotent postnatal stem cells from human periodontal ligament. Lancet 364: 149-155, 2004.

24. Zhu M, Kohan E, Bradley J, Hedrick M, Benhaim P and Zuk P: The effect of age on osteogenic, adipogenic and proliferative potential of female adipose-derived stem cells. J Tissue Eng Regen Med 3: 290-301, 2009.

25. Aldahmash A, Zaher W, Al-Nbaheen M and Kassem M: Human stromal (mesenchymal) stem cells: Basic biology and current clinical use for tissue regeneration. Ann Saudi Med 32: 68-77, 2012.

26. Chen FP, $\mathrm{Hu} \mathrm{CH}$ and Wang KC: Estrogen modulates osteogenic activity and estrogen receptor mRNA in mesenchymal stem cells of women. Climacteric 16: 154-160, 2013.

27. Morishita M, Yamamura T, Bachchu MA, Shimazu A and Iwamoto Y: The effects of oestrogen on osteocalcin production by human periodontal ligament cells. Arch Oral Biol 43: 329-333, 1998.

28. Morishita M, Yamamura T, Shimazu A, Bachchu AH and Iwamoto Y: Estradiol enhances the production of mineralized nodules by human periodontal ligament cells. J Clin Periodontol 26: 748-751, 1999.

29. Mamalis A, Markopoulou C, Lagou A and Vrotsos I: Oestrogen regulates proliferation, osteoblastic differentiation, collagen synthesis and periostin gene expression in human periodontal ligament cells through oestrogen receptor beta. Arch Oral Biol 56: 446-455, 2011.

30. Liang L, Yu JF, Wang Y, Wang G and Ding Y: Effect of estrogen receptor beta on the osteoblastic differentiation function of human periodontal ligament cells. Arch Oral Biol 53: 553-557, 2008.

31. Cao M, Shu L, Li J, Su J, Zhang W, Wang Q, Guo T and Ding Y: The expression of estrogen receptors and the effects of estrogen on human periodontal ligament cells. Methods Find Exp Clin Pharmacol 29: 329-335, 2007.

32. Zhang B, Li Y, Zhou Q and Ding Y: Estrogen deficiency leads to impaired osteogenic differentiation of periodontal ligament stem cells in rats. Tohoku J Exp Med 223: 177-186, 2011.

33. Liao S, Wang W, Uo M, Ohkawa S, Akasaka T, Tamura K, Cui F and Watari F: A three-layered nano-carbonated hydroxyapatite/collagen/PLGA composite membrane for guided tissue regeneration. Biomaterials 26: 7564-7571, 2005.

34. Liu HC, e LL, Wang DS, Su F, Wu X, Shi ZP, Lv Y and Wang JZ: Reconstruction of alveolar bone defects using bone morphogenetic protein 2 mediated rabbit dental pulp stem cells seeded on nano-hydroxyapatite/collagen/poly(L-lactide). Tissue Eng Part A 17: 2417-2433, 2011.
35. Yu SJ, Liu HC, Ling-Ling E, Wang DS and Zhu GX: Proliferation and differentiation of osteoblasts from the mandible of osteoporotic rats. Exp Biol Med (Maywood) 237: 395-406, 2012.

36. Gay IC, Chen S and MacDougall M: Isolation and characterization of multipotent human periodontal ligament stem cells. Orthod Craniofac Res 10: 149-160, 2007.

37. Zheng W, Wang S, Ma D, Tang L, Duan Y and Jin Y: Loss of proliferation and differentiation capacity of aged human periodontal ligament stem cells and rejuvenation by exposure to the young extrinsic environment. Tissue Eng Part A 15: 2363-2371, 2009.

38. E LL, Xu LL, Wu X, Wang DS, Lv Y, Wang JZ and Liu HC: The interactions between rat-adipose-derived stromal cells, recombinant human bone morphogenetic protein-2, and beta-tricalcium phosphate play an important role in bone tissue engineering. Tissue Eng Part A 16: 2927-2940, 2010.

39. Barrilleaux B, Phinney DG, Prockop DJ and O'Connor KC: Review: Ex vivo engineering of living tissues with adult stem cells. Tissue Eng 12: 3007-3019, 2006.

40. Caplan AI: Adult mesenchymal stem cells for tissue engineering versus regenerative medicine. J Cell Physiol 213: 341-347, 2007.

41. Eberli D and Atala A: Tissue engineering using adult stem cells. Methods Enzymol 420: 287-302, 2006.

42. Chang PC, Chien LY, Chong LY, Kuo YP and Hsiao JK: Glycated matrix up-regulates inflammatory signaling similarly to Porphyromonas gingivalis lipopolysaccharide. J Periodontal Res 48: 184-193, 2013.

43. Fan JZ, Wang Y, Meng Y, Li GW, Chang SX, Nian H and Liang YJ: Panax notoginseng saponins mitigate ovariectomy-induced bone loss and inhibit marrow adiposity in rats. Menopause 22: 1343-1350, 2015.

44. Lee JH, Baek HR, Lee KM, Zheng GB, Shin SJ and Shim HJ: Effects of ovariectomy and corticosteroid induced osteoporosis on the osteoinductivity of rhBMP-2 in a segmental long-bone defect model. Tissue Eng Part A 21: 2262-2271, 2015.

45. Fang J, Yang L, Zhang R, Zhu X and Wang P: Are there differences between Sprague-Dawley and Wistar rats in long-term effects of ovariectomy as a model for postmenopausal osteoporosis? Int J Clin Exp Pathol 8: 1491-1502, 2015.

46. Liu Y, Wang L, Liu S, Liu D, Chen C, Xu X, Chen X and Shi S: Transplantation of SHED prevents bone loss in the early phase of ovariectomy-induced osteoporosis. J Dent Res 93: 1124-1132, 2014.

47. Bressan E, Ferroni L, Gardin C, Pinton P, Stellini E, Botticelli D, Sivolella $S$ and Zavan B: Donor age-related biological properties of human dental pulp stem cells change in nanostructured scaffolds. PLoS One 7: e49146, 2012.

48. Sato C, Iso Y, Mizukami T, Otabe K, Sasai M, Kurata M, Sanbe T, Sekiya I, Miyazaki A and Suzuki H: Fibroblast growth factor-23 induces cellular senescence in human mesenchymal stem cells from skeletal muscle. Biochem Biophys Res Commun 470: 657-662, 2016.

49. Hong CJ, Park H and Yu SW: Autophagy for the quality control of adult hippocampal neural stem cells. Brain Res: March 9, 2016 (Epub ahead of print).

50. Takashima Y, Terada M, Udono M, Miura S, Yamamoto J and Suzuki A: Suppression of let-7b and miR-125a/b maturation by Lin $28 \mathrm{~b}$ enables maintenance of stem cell properties in hepatoblasts. Hepatology: March 17, 2016 (Epub ahead of print).

51. Larijani B, Arjmand B, Ahmadbeigi N, Falahzadeh K, Soleimani M, Sayahpour FA and Aghayan HR: A simple and cost-effective method for isolation and expansion of human fetal pancreas derived mesenchymal stem cells. Arch Iran Med 18: 770-775, 2015.

52. Tong L, Zhou J, Rong L, Seeley EJ, Pan J, Zhu X, Liu J, Wang Q, Tang X, Qu J, et al: Fibroblast growth factor-10 (FGF-10) mobilizes lung-resident mesenchymal stem cells and protects against acute lung injury. Sci Rep 6: 21642, 2016

53. Drowley L, Koonce C, Peel S, Jonebring A, Plowright AT, Kattman SJ, Andersson H, Anson B, Swanson BJ, Wang QD and Brolen G: Human induced pluripotent stem cell-derived cardiac progenitor cells in phenotypic screening: A transforming growth factor- $\beta$ type 1 receptor kinase inhibitor induces efficient cardiac differentiation. Stem Cells Transl Med 5: 164-174, 2016.

54. Tuganbekova S, Gaipov A, Turebekov Z, Saparbayev S, Shaimardanova G, Popova N, Taubaldiyeva Z, Serebrennikova D and Trimova R: Fetal renal stem cell transplant in nephrotic and nonnephrotic glomerulonephritis with stage 2-4 chronic kidney disease: Potential effect on proteinuria and glomerular filtration rate. 13 (Suppl 3): 156-159, 2015. 